\title{
Lebensstil und kardiovaskuläre Gesundheit - wie schädlich sind E-Zigaretten und Shisha-Rauchen?
}

\author{
Lifestyle and Cardiovascular Health - \\ How Harmful are E-cigarettes and Shisha Smoking?
}

(c) (ㄱ)(ㅇ)

\begin{abstract}
Autoren
Institute

1 Zentrum für Kardiologie, Kardiologie I, Universitätsmedizin der Johannes Gutenberg-Universität Mainz, Mainz, Deutschland

2 Deutsches Zentrum für Herz-Kreislauf-Forschung (DZHK), Standort Rhein-Main, Mainz, Deutschland
\end{abstract}

Marin Kuntic $^{1(\mathbb{D})}$, Omar Hahad ${ }^{1,2(\mathbb{D})}$, Thomas Münzel ${ }^{1,2}{ }^{\mathbb{D}}$, Andreas Daiber $^{1,2}$, $^{\mathbb{D}}$

\section{Schlüsselwörter}

E-Zigarettendampfen, Shisha-Rauchen, Endothelfunktion, oxidativer Stress, Entzündung

Key words

E-cigarette vaping, shisha smoking, endothelial function, oxidative stress, inflammation

Bibliografie

Aktuel Kardiol 2021; 10: 537-542

DOI 10.1055/a-1545-3107

ISSN 2193-5203

(C) 2021. The Author(s).

This is an open access article published by Thieme under the terms of the Creative Commons Attribution-NonDerivative-NonCommercial-License, permitting copying and reproduction so long as the original work is given appropriate credit. Contents may not be used for commercial purposes, or adapted, remixed, transformed or built upon. (https://creativecommons.org/licenses/by-nc-nd/4.0/).

Georg Thieme Verlag KG, Rüdigerstraße 14,

70469 Stuttgart, Germany

Korrespondenzadresse

Prof. Andreas Daiber

Zentrum für Kardiologie, Kardiologie I

Universitätsmedizin der Johannes Gutenberg-Universität

Mainz

Langenbeckstraße 1

55131 Mainz, Deutschland

daiber@uni-mainz.de

\section{ZUSAMMENFASSUNG}

Tabakrauchen ist ein Hauptauslöser chronischer nicht übertragbarer Krankheiten und ein Risikofaktor für kardiovaskuläre und pulmonale Erkrankungen. Obwohl der weltweite Tabak- konsum während der letzten 2 Jahrzehnte reduziert wurde, zeigt der Gebrauch von E-Zigaretten und Wasserpfeifen (Shisha) eine pandemische Zunahme mit hohem Anteil jüngerer Anwender. E-Zigaretten sind keine komplett schadensfreie Alternative zu herkömmlichen Tabakzigaretten, auch weil die zahlreichen Geschmacksaromen und die einfache/schnelle Verwendung eine steigende Zahl von abhängigen Anwendern hervorbringt. E-Zigaretten sind nicht unbedingt geeignet, die Nikotinabhängigkeit hinter sich zu lassen. Diese Arbeit bietet eine Übersicht über die kardiovaskulären Gesundheitsauswirkungen des Shisharauchens und E-Zigaretten-Dampfens mit Fokus auf die negativen Effekte auf die Endothelfunktion. Die schädlichen biologischen Auswirkungen der toxischen Inhaltsstoffe dieser Produkte werden vor allem im Hinblick auf oxidativen Stress und Entzündungsreaktionen diskutiert. Abschließend wird der aktuelle Stand hinsichtlich der Empfehlungen, gesetzlichen Regelungen und kommerziellen Werbung kurz zusammengefasst.

\section{ABSTRACT}

Tobacco smoking is a major trigger of chronic non-communicable diseases and a risk factor for cardiovascular and lung disease. Whereas global tobacco use was reduced during the last two decades, mostly due to a reduction of female smokers, the use of E-cigarettes and waterpipes (shisha) shows a pandemic growth, especially due to a lower starting age of users. In contrast to marketing arguments E-cigarettes are not a harm-free alternative to tobacco cigarettes. The young starting age, the numerous flavors and the easy/quick usage creates a growing number of addicted users. This also contradicts the main selling argument that E-cigarettes help quitting nicotine addiction. The present review provides an overview on health effects of shisha smoking and E-cigarette vaping with focus on negative effects on endothelial function and cardiovascular health. The adverse effects of the toxic constituents of these products on different biological pathways will be discussed, especially regarding oxidative stress and inflammatory cascades. Finally, the current state-of-the-art of recommendations, regulation and advertising will be briefly summarized. 


\section{WAS IST NEU/WICHTIG?}

Alle Tabakprodukte zusammen (ohne E-Zigaretten) sorgen laut der Weltgesundheitsorganisation (WHO) für jährlich 8 Millionen Todesfälle. Während das Tabakrauchen in den letzten 2 Jahrzehnten generell zurückgegangen ist, füllen alternative Genussmittel wie E-Zigaretten oder Wasserpfeifen diese Lücke. Es ist wichtig hervorzuheben, dass diese alternativen Genussmittel auch Gesundheitsrisikofaktoren darstellen und keinesfalls geeignet sind, um die Nikotinabhängigkeit zu überwinden. Da das Einstiegsalter bei E-Zigaretten und Wasserpfeifen niedriger liegt als bei klassischen Tabakzigaretten und auch Gelegenheitsanwender durch attraktive Aromastoffe und die einfache Anwendung angelockt werden, muss man zukünftig mit einer neuen Generation von Abhängigen rechnen. Eine große Gefahr liegt darin, dass vor allem E-Zigaretten ohne langjährige Erfahrungen hinsichtlich der gesundheitlichen Risiken auf breiter Front für den Markt zugelassen wurden. Sowohl präklinische Studien als auch klinische Studien sind dringend erforderlich, um die gesundheitlichen Risiken besser abschätzen zu können.

\section{Einleitung}

Der 2015 erschienene Bericht der Weltgesundheitsorganisation (WHO) zu den globalen Entwicklungen des Tabakrauchens beginnt mit dem Satz „Tabak ist eine legale Droge, die viele ihrer Nutzer tötet, wenn man sie genau so anwendet wie vom Hersteller angegeben“ [1]. Im Jahr 2019 berichtete die WHO, dass Tabakkonsum rund die Hälfte seiner Anwender umbringt und alle Tabakprodukte zusammen (ohne E-Zigaretten) für weltweit über 8 Millionen Todesfälle verantwortlich sind, wovon ca. 7 Millionen auf den direkten Tabakkonsum zurückzuführen sind und rund 1,2 Millionen auf das Passivrauchen [2]. Damit steht der Tabakkonsum an erster Stelle der vermeidbaren Gesundheitsrisiken und rangiert auch seit Jahrzehnten auf den ersten 10 Plätzen aller Risikofaktoren für die globale Mortalität und Morbidität, in einer der aktuellsten Global-Burden-of-Disease-Studien sogar auf dem zweiten Platz [3]. Obwohl der weltweite Tabakkonsum während der letzten 2 Jahrzehnte reduziert wurde, vor allem wegen einer Verringerung der Zahl weiblicher Raucher um mehr als 100 Millionen im Jahr 2018, wird dieser Rückgang durch neuartige Genussmittel wie E-Zigaretten und Wasserpfeifen (Shisha) kompensiert, die vor allem unter Jugendlichen sehr beliebt sind ( $\mathbf{A b b}$. 1) [2, 4].

Die Beliebtheit der E-Zigaretten und Wasserpfeifen bei der jüngeren Generation ist vor allem auf die zahlreichen Aromastoffe sowie die suggerierte gesundheitliche Sicherheit zurückzuführen, wobei Letzteres unter kritischer Betrachtung ein Trugschluss ist [5]. Der E-Zigaretten-Konsum hat sich in den USA zwischen 2011 und 2015 verneunfacht, und die weltweiten Verkaufserlöse werden bis zum Jahr 2023 auf nahezu 27 Milliarden US Dollar geschätzt [4]. Auch die Prävalenz des Shisharauchens in der allgemeinen Bevölkerung liegt in den USA, Großbritannien und Deutschland bei durchschnittlich 20-25\% [4]. Obwohl die Gesundheitsrisiken für E-Zigaretten und Wasserpfeifen im Vergleich zu Tabakzigaretten allgemein als geringer angesehen werden, konnte im Rahmen klinischer Studien gezeigt werden, dass das Risiko für Schlaganfall, Herzinfarkt und koronare Herzkrankheit auch bei den alternativen Genussmitteln erhöht ist (Übersicht in [4]). So zeigen aktuellere Metaanalysen und Primärstudien Risikoerhöhungen von 89, 134 und 194\% für koronare Herzkrankheiten als Konsequenz von E-Zigaretten, Tabakzigaretten und Shisha (in aufsteigender Reihenfolge, basierend auf Studien mit unterschiedlichem Qualitätslevel), wobei bezüglich des Schlaganfallrisikos Erhöhungen von $61 \%$ und $335 \%$ als Konsequenz von Tabakzigaretten und Shisha ermittelt wurden, während dies nicht für E-Zigaretten beobachtet werden konnte (wieder basierend auf Studien mit stark unterschiedlichem Qualitätslevel) [4].

\section{KURZGEFASST}

Die Summe aller Tabakprodukte (ohne E-Zigaretten) ist weltweit für jährlich 8 Millionen Todesfälle verantwortlich. Tabakrauchen rangiert seit Jahrzehnten auf den ersten 10 Plätzen auf der Liste aller Gesundheitsrisikofaktoren. Der Rückgang des Tabakrauchens in den letzten Jahrzehnten wurde teilweise durch neue Genussmittel wie E-Zigaretten oder Wasserpfeifen kompensiert.

\section{Schadstoffe und Pathomechanismen}

Obwohl die Pathomechanismen im Gegensatz zum Tabakzigarettenrauchen für den E-Zigaretten-Konsum weniger gut aufgeklärt sind und die klinische Datenlage sowohl für den E-Zigaretten-Konsum als auch das Shisharauchen deutlich schlechter ist als für den Konsum von Tabakzigaretten, haben sich als zentrale Pathomechanismen die Bildung reaktiver Sauerstoffspezies (oxidativer Stress), Entzündungsreaktionen und die endotheliale Dysfunktion etabliert. Für den Konsum von Tabakzigaretten wurde bereits im Jahr 1993 eine Verschlechterung der endothelvermittelten Gefäßvasodilatation gezeigt [6], die durch die Gabe von Vitamin C aufgehoben werden konnte (als indirekter Beweis für die Beteiligung von oxidativem Stress) [7]. Vor allem für E-Zigaretten-Konsum konnten sowohl bei Probanden [8] als auch im Tiermodell unter chronischer [9] und kurzzeitiger Exposition [10, 11] mit Zigarettendampf eine massive Verschlechterung der Endothelfunktion und Bluthochdruck nachgewiesen werden. Anhand einer Mausstudie konnten der oxidative Stress und Entzündungsreaktionen als zentrale Pathomechanismen für eine endotheliale Dysfunktion und die kardiovaskulären Komplikationen identifiziert werden [10]. Auch für Shisharauchen wurden ähnliche klinische und tierexperimentelle Befunde publiziert (Überblick in [4]). Sowohl die periphere als auch koronare endotheliale Dysfunktion (gemessen mittels flussabhängiger Dilatation bzw. Acetylcholin-induzierter paradoxer Vasokonstriktion in den Koronarien) wird als frühes Korrelat einer subklinischen atherosklerotischen Veränderung angesehen und hat laut zahlreichen klinischen Studien prognostische Aussagekraft hinsichtlich des zukünftigen kardiovaskulären Risikos [4].

Nikotin hat zwar eine zentrale Bedeutung für das Suchtpotenzial des Rauchens bzw. Dampfens, jedoch nur eine geringe gesundheitsschädliche Bedeutung. Die gesundheitsschädlichen Wir- 
US-Raucherquote fällt auf ein Rekordtief

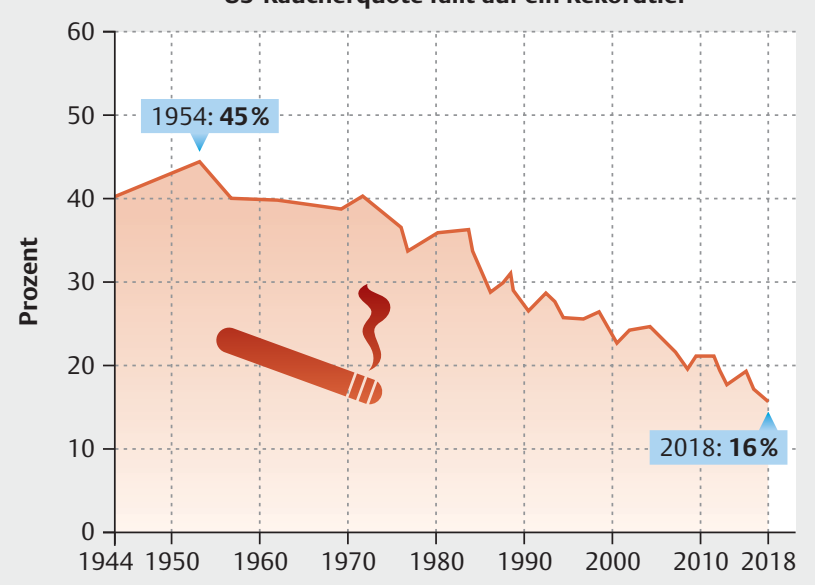

US-Teenager präferieren E-Zigaretten

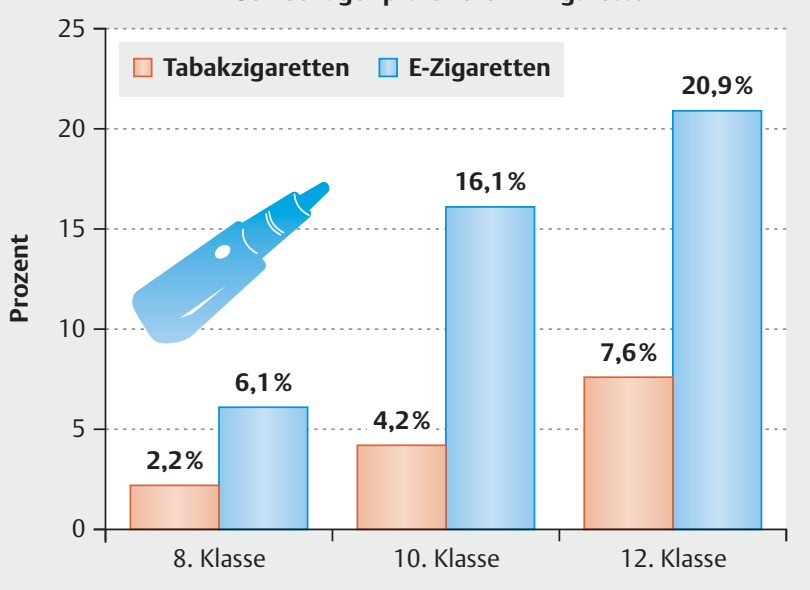

- Abb. 1 Obwohl die Raucherquote in den USA seit Jahrzehnten rückläufig ist, wird dieser Gesundheitsvorteil durch die steigende Zahl der E-Zigaretten-Konsumenten teilweise kompensiert, da vor allem jüngere Schüler und Studenten zur E-Zigarette greifen. Daten nach [20, 21]

kungen des Tabakrauchs oder E-Zigaretten-Dampfs sind auf die bekannten toxischen Substanzen im Rauch bzw. Dampf zurückzuführen, die bei der Verbrennung bzw. Erhitzung entstehen ( $\vee$ Tab. 1). Eine gute Übersicht über die bekannten toxischen Substanzen im Tabakzigarettenrauch bietet Referenz [12] und Referenz [13] für E-Zigaretten-Dampf und Liquid, die auch unter Berücksichtigung der Besonderheiten von Shisharauch (dort sind die Verbrennungstemperaturen niedriger als bei Tabakzigaretten) in Referenz [4] und dem zugehörigen Online-Supplement sehr ausführlich diskutiert werden. Während Shisharauch ähnliche Schadstoffe wie Tabakzigarettenrauch enthält, die durch die Verbrennung von Tabak entstehen, unterscheidet sich das Profil der toxischen Substanzen im E-Zigaretten-Dampf signifikant, da dieser nicht durch Verbrennung, sondern durch Verdampfung bei deutlich geringeren Temperaturen stattfindet. Die Basis für den E-Zigaretten-Dampf bildet nicht der Tabak, sondern das sogenannte Liquid, ein Gemisch, das aus den Hauptbestandteilen Polyethylenglykol und Glycerin besteht, dem unterschiedliche Konzentrationen Nikotin und sonstige Zusatzstoffe wie Aromastoffe zugesetzt werden. Die Toxizität des Tabakrauchs wird vor allem durch Übergangsmetalle, Kohlenmonoxid und andere toxische Gase, NNitrosamine, fester Feinstaub, volatile organische Bestandteile (VOCs, z. B. Benzol) und polyzyklische aromatische Kohlenwasserstoffe (PAHs, z. B. Benzo[a]pyren) bestimmt ( $\triangleright$ Tab. 1). Die maßgeblichen Schadstoffe in E-Zigaretten-Dampf sind reaktive Aldehyde wie Formaldehyd und Acrolein und in geringerem Maße Über- gangsmetalle. N-Nitrosamine und VOCs konnten dagegen nur in Spuren nachgewiesen werden.

Die oben erwähnten Schadstoffe im E-Zigaretten-Dampf induzieren hauptsächlich oxidativen Stress und Entzündungsreaktionen und nachfolgend eine endotheliale Dysfunktion, Progression der Atherosklerose und ein erhöhtes Risiko für kardio(zerebro)vaskuläre Erkrankungen. Anhand einer Mausstudie konnten die im E-Zigaretten-Dampf enthaltenen toxischen Aldehyde wie Formaldehyd und Acrolein als ursächlich für den oxidativen Stress und Entzündungsreaktionen sowie die nachfolgende endotheliale Dysfunktion identifiziert werden [10]. Wie in $>$ Tab. 1 dargestellt, gibt es eine Überschneidung hinsichtlich der Schadstoffe in E-ZigarettenDampf und dem Rauch von Tabakzigaretten und Wasserpfeifen, sprich auch die Pathomechanismen zeigen eine Überlappung und oxidativen Stress, Entzündungsreaktionen und Gefäßdysfunktion als zentrale Mediatoren [4]. Die im Tabakrauch enthaltenen zusätzlichen Schadstoffe wie Feinstaub, toxische Gase, VOCs, PAHs und Übergangsmetalle wurden allesamt in der Vergangenheit ebenfalls als Auslöser für oxidativen Stress, Entzündungsreaktionen und Gefäßdysfunktion beschrieben. Die gesundheitsschädliche Bedeutung dieser Schadstoffe im Tabakrauch wurde durch die signifikante Verringerung der tabakassoziierten kardiovaskulären Ereignisse und Hospitalisierung nach Einführung des Nichtraucherschutzgesetzes im Jahr 2003 unterstrichen [14, 15], das hauptsächlich zum Ziel hatte, die Nichtraucher vor den Auswirkungen des Passivrauchens zu schützen. 
- Tab. 1 Vergeich der Schadstoffe, biologischen Schäden und Gesundheitsauswirkungen von Tabakrauch, Shisharauch und E-Zigaretten-Dampf. In der unteren Reihe sind die Gesundheitsauswirkungen dargestellt. Die Tabelle wurde aus Daten in [4] zusammengestellt.

\begin{tabular}{|c|c|c|c|}
\hline & Tabakrauchen & Shisharauchen & E-Zigaretten-Dampfen \\
\hline Hauptschadstoffe & $\begin{array}{l}\text { hohe Konzentrationen: } \\
\text { - Übergangsmetalle } \\
\text { - Kohlenmonoxid } \\
\text { - } \text { Aldehyde } \\
\text { - Nikotin } \\
\text { - N-Nitrosamine } \\
\text { - flüchtige organische Verbindungen } \\
\text { (VOCs) } \\
\text { mittlere Konzentrationen: } \\
\text { " fester Feinstaub } \\
\text { - polyzyklische aromatische Kohlen- } \\
\text { wasserstoffe (PAHs) }\end{array}$ & $\begin{array}{l}\text { hohe Konzentrationen: } \\
\text { - Übergangsmetalle } \\
\text { - Kohlenmonoxid } \\
\text { - fester Feinstaub } \\
\text { mittlere Konzentrationen: } \\
\text { - } \text { Aldehyde } \\
\text { - Nikotin } \\
\text { - N-Nitrosamine } \\
\text { - flüchtige organische Verbindungen } \\
\text { (VOCs) } \\
\text { - polyzyklische aromatische Kohlen- } \\
\text { wasserstoffe (PAHs) }\end{array}$ & $\begin{array}{l}\text { hohe Konzentrationen: } \\
\text { " Aldehyde } \\
\text { " Nikotin } \\
\text { mittlere Konzentrationen: } \\
\text { " Übergangsmetalle } \\
\text { niedrige Konzentrationen: } \\
\text { " N-Nitrosamine } \\
\text { - flüchtige organische Verbindungen } \\
\text { (VOCs) }\end{array}$ \\
\hline $\begin{array}{l}\text { molekulare Marker } \\
\text { für Schäden }\end{array}$ & 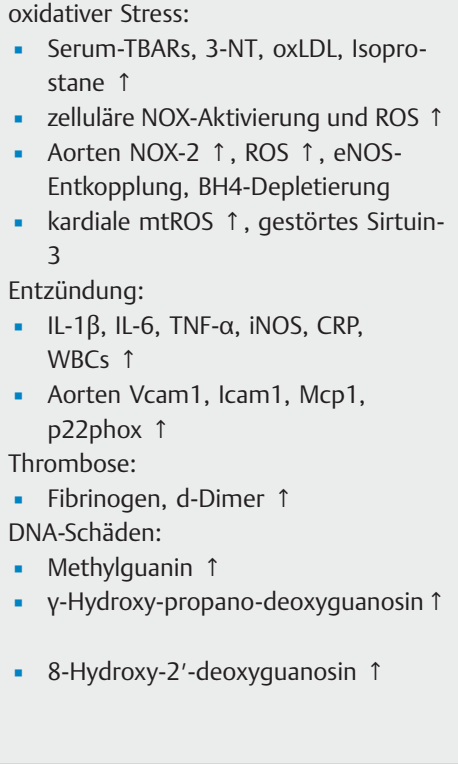 & 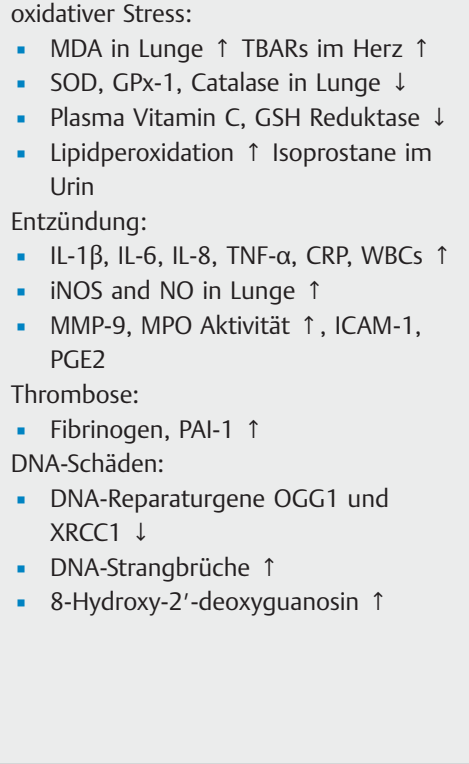 & 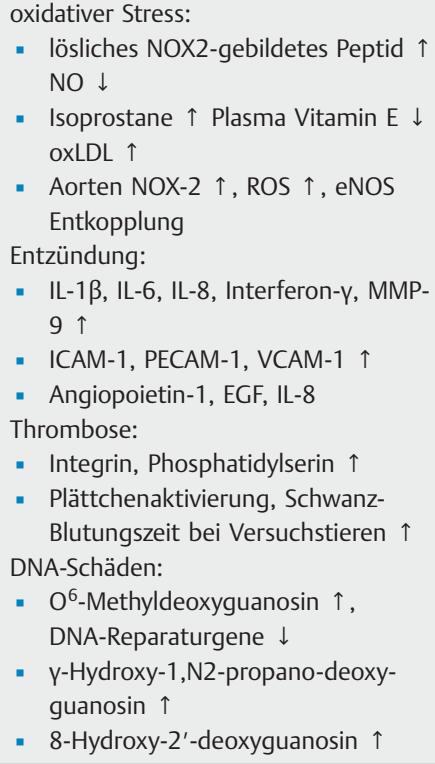 \\
\hline $\begin{array}{l}\text { assoziierte negative } \\
\text { Gesundheitseffekte }\end{array}$ & $\begin{array}{l}\text { - } \text { atherosklerotische Plaques } \\
\text { - } \text { zerebrale Aneurysmen } \\
\text { - } \text { gestlagenablagerung } \\
\text { - Hypertonie } \\
\text { - Emphysem } \\
\text { - } \text { mehr LDL und Gesamtcholesterol } \\
\text { - COPD, Krebs, ischämische Herz- } \\
\text { krankheit } \\
\text { - Dysregulation der zirkadianen } \\
\text { Rhythmik }\end{array}$ & $\begin{array}{l}\text { " } \quad \text { hörombores Herzgewicht (Troponin I und } \\
\text { B-Typ natriuretisches Peptid) } \\
\text { " COPD, Krebs, ischämische Herzkrank- } \\
\text { heit } \\
\text { " endotheliale Dysfunktion } \\
\text { " Dysregulation der zirkadianen Rhyth- } \\
\text { mik }\end{array}$ & $\begin{array}{l}\text { - Fibrose multipler Organe } \\
\text { - } \text { COPD, Krebs, ischämische Herz- } \\
\text { krankheit } \\
\text { - endotheliale Dysfunktion } \\
\text { - ischämische Hirnschäden } \\
\text { - Dysregulation der zirkadianen } \\
\text { Rhythmik }\end{array}$ \\
\hline
\end{tabular}

\section{KURZGEFASST}

E-Zigarettenkonsum induziert in Probanden und in Mäusen eine vergleichbare Schädigung der Endothelfunktion wie Tabakzigarettenrauch. Shisharauch verhält sich komplexer, da er hohe Konzentrationen Kohlenmonoxid enthalten kann, das vasodilatierend wirkt. Oxidativer Stress und Entzündungsreaktionen sind die Hauptmediatoren für die Gefäßschäden und werden durch ein komplexes Schadstoffgemisch im E-Zigaretten-Dampf und Tabakrauch induziert. Es ist wichtig festzuhalten, dass die kardiovaskulären Schä- den durch E-Zigaretten-Dampf und Tabakrauch nicht von Nikotin verursacht werden, sondern durch die bei der Erhitzung bzw. Verbrennung entstehenden toxischen Substanzen im Rauch bzw. Dampf. Nikotin hat zentrale Bedeutung für die Abhängigkeit und Suchtspirale. 


\section{Ist E-Zigaretten-Dampfen eine weniger schäd- liche Alternative zu Tabakzigarettenrauchen?}

Diese Frage ist nach derzeitiger begrenzter Datenlage klar mit einem „Ja“ zu beantworten. Zahlreiche klinische Studien zeigen, dass ein Wechsel von Tabakzigarette zu E-Zigarette mit einer schnellen (innerhalb eines Monats) und signifikanten Verbesserung der Endothelfunktion vergesellschaftet ist (Überblick in [4]). Mehrere klinische Studien zeigen einen eindeutigen Benefit (auch hinsichtlich des oxidativen Stresses) für den E-ZigarettenKonsum im Vergleich zum Tabakrauchen [16]. Auch ist allgemein anerkannt, dass die durch die Center for Disease Control (CDC), Food \& Drug Agency (FDA) und US State Health Departments berichteten dramatischen Lungenerkrankungen und Todesfälle von amerikanischen Bürgern im Zusammenhang mit dem Konsum von E-Zigaretten auf Zusätze wie Vitamin-E-Acetat und Tetrahydrocannabinol (THC) zurückzuführen waren. Demnach könnten E-Zigaretten zur Entwöhnung für Raucher eingesetzt werden, indem Schritt für Schritt die Nikotinkonzentration im Liquid verringert wird, um so ein „Ausschleichen“ des Tabakkonsums zu erzielen. Daten einer aktuellen Cochrane Metaanalyse deuten in diese Richtung [17], wohingegen eine andere Metaanalyse keine signifikanten Anzeichen für eine Raucherentwöhnung durch E-Zigaretten finden konnte [18].

Was aber berücksichtigt werden muss, ist, dass nach wie vor große langfristige klinische Studien fehlen, welche die Gesundheitsauswirkungen des E-Zigaretten-Konsums in der allgemeinen Bevölkerung untersuchen [19]. Analog fehlen Studien zu den Gesundheitsauswirkungen von Shishakonsum. Wichtig ist auch zu bedenken, dass, obwohl E-Zigaretten wahrscheinlich etwas weniger schädlich sind als Tabakzigaretten, das Einstiegsalter für E-Zigaretten-Konsum niedriger liegt und E-Zigaretten-Anwender oft auch Tabakzigaretten rauchen („Dual Users“); ferner kann man EZigaretten noch problemloser überall im öffentlichen Raum anwenden oder auch noch häufiger für nur wenige Sekunden nutzen („Only one Puff“). Vor allem die steigende Nutzung unter Jugendlichen könnte langfristig eine neue Generation der Abhängigen generieren und aufgrund des sehr frühen Einstiegs chronische Erkrankungen im fortgeschrittenen Lebensalter fördern, dadurch zu mehr verlorenen gesunden Lebensjahren (DALYs) führen und so die Gesamtgesundheitsbelastung der Bevölkerung steigern.

\section{KURZGEFASST}

E-Zigaretten scheinen nach aktueller Datenlage weniger schädlich zu sein als Tabakzigaretten. Es fehlen jedoch valide große klinische Studien. Aufgrund zusätzlicher Risiken der E-Zigaretten wie jüngeres Einstiegsalter oder intensivere Nutzung sollte der unter anderem von Public Health England und dem Royal College of Physicians propagierte Slogan, dass E-Zigaretten „95\% gesünder“ seien als Tabakzigaretten, keinesfalls ernst genommen werden.

\section{Gesetzliche Lage und wissenschaftliche Empfehlungen}

Die American Heart Association warnt wiederholt vor dem E-Zigaretten-Konsum. Sowohl E-Zigarette als auch Shisha induzieren auf pathomechanistischer Ebene oxidativen Stress und Entzündungsreaktionen. Für E-Zigaretten wurde auch die Entstehung einer endothelialen Dysfunktion in Probanden und im Tiermodell beobachtet. Obwohl E-Zigaretten nach aktueller Datenlage wahrscheinlich weniger schädlich sind als Tabakzigaretten, muss festgehalten werden, dass große randomisierte klinische Studien fehlen. Dazu kommt das sinkende Einstiegsalter und häufigere Nutzung durch die Anwender. Um die Bevölkerung, vor allem die Jugend, vor diesen neuen Gefahren besser zu schützen, sollte die Gesetzgebung deren strikte Regulierung überwachen (Überblick in [4]). Mittlerweile ist der Verkauf von E-Zigaretten in zahlreichen Ländern gesetzlich untersagt, dies betrifft Indien, Australien und Mexiko sowie zahlreiche südamerikanische und arabische Länder (rund 35\% der Weltbevölkerung). In einem weiteren Teil der Welt, inklusive Europa, USA, Kanada und China werden E-Zigaretten (Verkauf, Inhaltsstoffe etc.) stark vom Gesetzgeber reguliert (rund 35\% der Weltbevölkerung). Im restlichen Teil der Welt werden E-Zigaretten ohne jegliche Auflagen auf den Markt gebracht. Ein ähnlicher Flickenteppich besteht für die kommerzielle Werbung für Tabakprodukte und E-Zigaretten - in rund der Hälfte der europäischen Länder (inklusive Deutschland) ist die Tabakwerbung erlaubt.

\section{Fazit}

Der Konsum von Tabakprodukten führt jährlich zu mehr als 8 Millionen vermeidbaren Todesfällen weltweit, wobei ein Großteil kardiovaskuläre Ursachen hat. Trotz der sinkenden Zahlen der weltweiten Tabakzigarettenraucher in den letzten Jahrzehnten werden die gesundheitlichen Vorteile teilweise durch neue Genussmittel kompensiert. E-Zigaretten-Konsum und Shisharauchen erhöhen das Risiko für Herz-Kreislauf-Erkrankungen, Schlaganfälle und chronische Lungenerkrankungen. Abschließend muss festgehalten werden, dass, ungeachtet der Risiken von E-Zigaretten und Shisha als „Einstiegsdroge“ für junge Menschen, regelmäßiges Tabakrauchen schädlicher ist als E-Zigaretten-Dampfen oder gelegentliches Shisharauchen, vor allem im Hinblick auf die Inzidenz von Krebserkrankungen, aber auch hinsichtlich der kardiovaskulären Risiken.

\section{Fördermittel}

Mainzer Herz 17 und 21 | Stiftung Mainzer Herz | http://www.herzstiftung-mainzer-herz.de/herzstiftung/startseite/uebersicht.html

Standort Rhein-Main, Partner Mainz | Deutsches Zentrum für HerzKreislaufforschung | https://dzhk.de/

Research Consortium "Novel and neglected cardiovascular risk factors" | Boehringer Ingelheim Fonds | https://www.boehringer-ingelheim-stiftung.de/was-wir-foerdern/initiativen-fuer-die-region/initiativen-an-der-unimedizin-mainz.html 
Interessenkonflikt

Die Autorinnen/Autoren geben an, dass kein Interessenkonflikt besteht.

Literatur

[1] World Health Organization. WHO global report on trends in prevalence of tobacco smoking 2015. Geneva: WHO; 2015. Im Internet (Stand: 07.01.2020): https://apps.who.int/iris/bitstream/handle/10665/156262/ 9789241564922_eng.pdf?sequence $=1$

[2] World Health Organization. WHO global report on trends in prevalence of tobacco use 2000-2025, third edition. Geneva: WHO; 2019. Im Internet (Stand: 07.01.2020): https://www.who.int/publications-detail/whoglobal-report-on-trends-in-prevalence-of-tobacco-use-2000-2025-thirdedition

[3] Cohen AJ, Brauer M, Burnett R et al. Estimates and 25-year trends of the global burden of disease attributable to ambient air pollution: an analysis of data from the Global Burden of Diseases Study 2015. Lancet 2017; 389: 1907-1918. doi:10.1016/S0140-6736(17)30505-6

[4] Munzel T, Hahad O, Kuntic M et al. Effects of tobacco cigarettes, e-cigarettes, and waterpipe smoking on endothelial function and clinical outcomes. Eur Heart J 2020; 41: 4057-4070. doi:10.1093/eurheartj/ehaa4 60

[5] Eissenberg T, Bhatnagar A, Chapman S et al. Invalidity of an Oft-Cited Estimate of the Relative Harms of Electronic Cigarettes. Am J Public Health 2020; 110: 161-162. doi:10.2105/AJPH.2019.305424

[6] Celermajer DS, Sorensen KE, Georgakopoulos D et al. Cigarette smoking is associated with dose-related and potentially reversible impairment of endothelium-dependent dilation in healthy young adults. Circulation 1993; 88: 2149-2155. doi:10.1161/01.cir.88.5.2149

[7] Heitzer T, Just $\mathrm{H}$, Munzel T. Antioxidant vitamin C improves endothelial dysfunction in chronic smokers. Circulation 1996; 94: 6-9. doi:10.1161/ 01.cir.94.1.6

[8] Vlachopoulos C, loakeimidis N, Abdelrasoul M et al. Electronic Cigarette Smoking Increases Aortic Stiffness and Blood Pressure in Young Smokers. J Am Coll Cardiol 2016; 67: 2802-2803. doi:10.1016/j.jacc.2016.03.569

[9] Olfert IM, DeVallance E, Hoskinson $\mathrm{H}$ et al. Chronic exposure to electronic cigarettes results in impaired cardiovascular function in mice. J Appl Physiol 2018; 124: 573-582. doi:10.1152/japplphysiol.00713.201 7
[10] Kuntic M, Oelze M, Steven S et al. Short-term e-cigarette vapour exposure causes vascular oxidative stress and dysfunction: evidence for a close connection to brain damage and a key role of the phagocytic NADPH oxidase (NOX-2). Eur Heart J 2020; 41: 2472-2483. doi:10.1093/ eurheartj/ehz772

[11] Rao P, Liu J, Springer ML. JUUL and Combusted Cigarettes Comparably Impair Endothelial Function. Tob Regul Sci 2020; 6: 30-37. doi:10.180 01/TRS.6.1.4

[12] Rodgman A, Perfetti T. The Chemical Components of Tobacco and Tobacco Smoke. Boca Raton: CRC Press; 2013. . doi:10.1201/b13973

[13] Charlotta P. A systematic review of health effects of electronic cigarettes. Document prepared for the World Health Organization 2015. Geneva: WHO; 2016.

[14] Sargent JD, Demidenko E, Malenka DJ et al. Smoking restrictions and hospitalization for acute coronary events in Germany. Clin Res Cardiol 2012; 101: 227-235. doi:10.1007/s00392-011-0385-1

[15] Anonymous. Rauchverbote haben in Deutschland Tausende Leben gerettet. Im Internet (Stand: 17.10.2021): https://www.sueddeutsche.de/gesundheit/tabakkonsum-rauchverbote-haben-in-deutschland-tausendeleben-gerettet-1.1308347

[16] Carnevale R, Sciarretta S, Violi F et al. Acute Impact of Tobacco vs Electronic Cigarette Smoking on Oxidative Stress and Vascular Function. Chest 2016; 150: 606-612. doi:10.1016/j.chest.2016.04.012

[17] Hartmann-Boyce J, McRobbie H, Lindson N et al. Electronic cigarettes for smoking cessation. Cochrane Database Syst Rev 2021(04): CD010216. doi:10.1002/14651858.CD010216.pub5

[18] Pound CM, Zhang JZ, Kodua AT et al. Smoking cessation in individuals who use vaping as compared with traditional nicotine replacement therapies: a systematic review and meta-analysis. BMJ Open 2021; 11 : e044222. doi:10.1136/bmjopen-2020-044222

[19] Munzel T, Kuntic M, Steven $S$ et al. Is vaping better than smoking cigarettes? Eur Heart J 2020; 41: 2612-2614. doi:10.1093/eurheartj/ehaa2 67

[20] Statista. U.S. Smoking Rate Falls To Record Low. 2018. Im Internet (Stand: 18.10.2021): https://www.statista.com/chart/14879/us-smoking-rate-falls-to-record-low/

[21] Statista. U.S. Teens More Likely to Use E-Cigarettes than Cigarettes. 2019. Im Internet (Stand: 18.10.2021): https://www.statista.com/chart/ 18633/use-of-cigarettes-and-e-cigarettes-among-us-teens/ 